\title{
Sustainable safety management: incident management as a cornerstone for a successful safety culture
}

\author{
B. Freibott \\ SAP Germany $A G$ \& Co. KG, Germany
}

\begin{abstract}
Most managers agree that protecting people and the environment is all about prevention. But with today's tight budgets and cutbacks, the environmental and safety professions must choose wisely where to invest in prevention programs that will yield the best results. Knowing the details about your organization's incident history is one key component to improving and maintaining low incident rates and their associated injuries, illnesses, and environmental releases. By regulation, most of us have to report our incidents in accordance with our region's regulatory processes, and the Environment, Health, and Safety (EHS) management systems do that very nicely. However, positive gains in efficiency and lower costs are gained by going beyond compliance to a point where you are anticipating and intervening with any negative trends that a proactive modern EHS management system can help identify. Beyond environmental incidents and accidents, the term "incident management" implies that any observations about hazards or unsafe behaviors are reported and addressed before they become a costly incident.

Modern incident management and its applied tools must address various stakeholders with different perspectives, and varying degrees of computer proficiency. All stakeholders must be intuitively comfortable with the solution and find it easy to use for their purposes. The process of incident management must also ensure that all data is provided, approved, and available for reporting. All corrective actions must be tracked, and escalations automatically delivered if they are overdue. A modern incident management system is the central communication platform that provides the right tools and the right information for all the involved people and stakeholders.
\end{abstract}

Keywords: EHS management system, incident management, incident prevention, 
industrial hygiene, information technology (IT), occupational health and safety, process design, project management, safety culture.

\section{Introduction}

"The Basics of Incident Management - 200 Years of Safety Management". Actually, incident management is nothing new at all. Historically seen, the birth year of occupational health and safety is probably 1811, when DuPont - a producer of explosives along the Brandywine River in the United States established the first safety rules for their operations in their production plants. "All kind of play or disorderly fun is prohibited," read a notice posted by the company's French immigrant founder E.I. du Pont de Nemours on New Year's Day, 1811. Seven years later he banned alcohol from the site after an explosion killed 40 people and injured his wife Sophie. The accident was attributed to a DuPont foreman's drinking.

Still, a series of major explosions in the early decades of the 19th century occurred, and drove the DuPont family to strive for more safety regulations and measures, and by these means to diminish the operative risk of their production plants and the personal risk for their employees.

As often is the case in occupational health and safety, major accidents and incidents have to happen to make things change. Consequently, the lessons learned from incidents were the main basis for evolutions in safety management. And for a great part of the industries around the world, this is also today's reality.

Nonetheless, for the next 100-150 years, it was considered an inevitable collateral effect of any operations that accidents would happen and that people would be injured.

The 20th century saw the improvement and spread of physical safety measure and procedures, and the last 50 years have seen the evolution of safety management systems as systematic approaches to safety.

In context of safety management systems, incident management has been one of the major triggers for improvement and changes. The objective was essentially this: something that has happened should never happen again, and everything should be done to prevent accidents from repeating, to diminish the danger to which employees are exposed, and to reduce the risk of operations.

This methodology in the beginning was bound to be reactive and was very far from becoming a proactive safety management. The next essential step toward a safer work environment was to better understand what the reasons are for accidents - to better grasp the nature of the causes of and the action chains that lead to accidents.

\section{The causes of incidents and accidents}

Certainly, the last 200 years of safety management have not been without effect. Safety regulations and safety management strongly diminished the number of incidents in many industries, and protective equipment and safety measures 
reduced the impact of accidents, leading to fewer accidents, less serious incidents, and fewer fatalities.

But still, incidents and accidents happen, and people still get hurt and sometimes die from accidents at work. Everybody knows the major incidents that have happened in the last years, with grave environmental consequences and with workers hurt or killed. The Deepwater Horizon oil rig catastrophe is just one prominent example. Beyond these major incidents with great publicity, the incident numbers are much diminished, but it also seems that on this diminished level they have reached a kind of low barrier.

So why do these incidents happen, though more and more companies strive for a zero-accident environment? The reason is not obvious, but a closer look can help: studies have shown that a great many accidents are not the consequence of a major danger or hazard, nor do they happen due to missing safety regulation or safety equipment. A great number, if not the majority, of accidents happen as the consequence of minor lapses, and usually of not just one lapse but the sequence of minor failures.

For example:

1. An employee is in a certain work area for the first time, and by chance he has not had the relevant safety instruction that is obligatory for that work area.

2. Due to the new work environment, he forgets to affix one of the safety hooks properly on his gear.

3. On that day, accidentally, the supervisor is not in place, and no replacement has been nominated yet.

4. The worker has not had the obligatory occupational health check, including a check for being free from dizziness.

Result: the worker becomes dizzy working in a high place, loses hold, slips out of the wrongly fixed safety hook, and falls to his death.

The important message of this hypothetical scenario is that none of the single lapses alone would have caused a fatal accident, but the combination of minor lapses creates a safety gap that can lead to major accidents.

A classical image for this phenomenon is the cheese slice model. The cheese slices represent protective layers, controls such as safety regulation and management, fit-for-work checks, supervision, protective equipment, and so forth. Only where these protective layers fail - holes in the cheese - is there a latent danger. And only when all layers fail, when there are holes or safety gaps along the stream of action, can an accident with major impact happen.

What does this mean for a proactive incident management?

The obvious message is: learning from real incidents and accidents is only a small part of what is needed. It is like chipping off little pieces from the top of an iceberg. It will have an effect, but the change will not be great. The first challenge is to get a better understanding of what is happening in the company, what is the reality.

This idea about "the reality" within a company with respect to safety, safe operations, and safe behavior is not entirely new. In 1931, A. W. Heinrich, an assistant superintendent of the Engineering and Inspection Division of Travelers Insurance Company, published his book Industrial Accident Prevention, which 


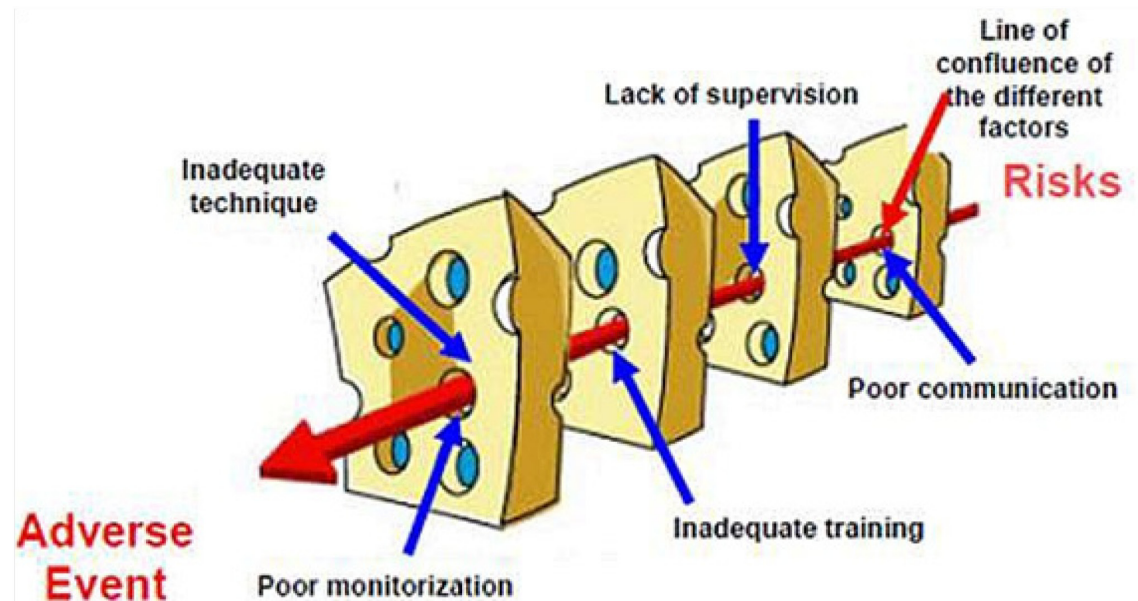

Figure 1: Cheese slice model.

included his empirical finding known as Heinrich's Law: that in a workplace, for every accident that causes a major injury, there are 29 accidents that cause minor injuries and 300 accidents that cause no injuries [1].

In 1969, Frank E. Bird Jr. drilled even deeper in a study of industrial accidents, during which he analyzed more than 1.7 million accidents reported by 297 companies [2]. The essential finding was that for every reported major injury (fatality, disability, lost time, and medical treatment) there were 9.8 reported minor injuries. For each major accident with lost time, there were around 15 accidents requiring medical treatment and 30.2 property damage accidents. Diving deeper during this extensive study, Bird found out that below those real accidents, there was a bottom layer of around 600 near misses or incidents that might have caused injuries or major accident. Overall these findings are usually depicted in a pyramid with a 1-10-30-600 ratio.

In 2003, a study by ConocoPhillips Marine [3] further demonstrated the large difference between serious accidents and near misses. The core message of the ConocoPhillips study was that for each single fatality there must be assumed a hidden bottom end of the pyramid of 300,000 at-risk behaviors. At-risk behaviors are defined as any activity that is not consistent with safety programs or with training on machinery - such as bypassing safety components on machinery, eliminating safety steps in the production process, and so forth.

The natural consequence is that proactive safety management and incident management cannot complacently analyze accidents and real occurrences but rather has to strive for transparency, creating visibility on what is below the surface. This means not just chipping off the top of the iceberg but really getting know the iceberg and tackling it from below. 


$$
\begin{array}{|c|c|c|}
1 \\
\text { Major } \\
\text { Injury } \\
29 \\
\text { Minor } \\
\text { Injury } \\
\mathbf{3 0 0} \\
\text { Incidents } \\
\text { (near miss) }
\end{array}
$$

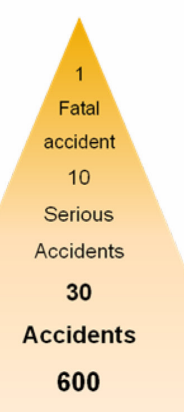

Incidents

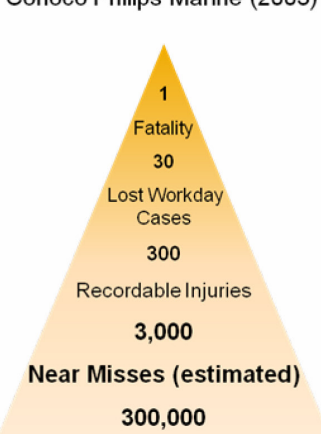

At-Risk Behaviors (estimated)

Figure 2: $\quad$ Heinrich pyramid and similar studies.

\section{Incident management as the principal pillar of preventive safety management}

As we have seen, incidents today are not the consequence of major dangers, but rather the result of chains of minor lapses. The first step toward an improvement is therefore to gather all information about what is happening within the company - to collect information on every kind of hazard or safety-related misconduct.

\subsection{Reporting of all kinds of safety-related incidents}

One of the first steps when setting up a proactive safety management is to define what kinds of incidents or occurrences are to be collected.

At the top of the list are still all incidents and accidents. It remains a core function of incident management to register all real incidents and accidents, create all the relevant reporting and statistics, execute incident investigation and root-cause analysis, trigger corrective actions and safety measures, and in this way make sure that the same things cannot happen again.

The next step after this core activity of incident management is to capture in some way all available information on all kinds of hazards that occur and that are not considered in the industrial hygiene and risk assessment processes. These can be physical hazards caused by assets, but also operational hazards caused by the operative processes or the way of doing things. These are the hazards that are in the nature of the plants, operations, assets, and activities of the company.

Further, there are the accidental hazards, such as latent dangers caused by malfunctions or damage of assets, or by safety gaps in the operations. Examples are a damaged electric plug, a damaged ladder, or a consumed safety rope.

After to the real incidents and accidents, and the real hazards, it is then important to collect all information about any kind of near misses or nearby accidents. Though these near misses do not have a real impact, they are just as 
valuable a source of information as real accidents and it is important to derive the right lessons from them. In other words, having just escaped the accident, one should make sure to prevent similar situations from happening.

A very important factor in this context is the human factor. Human behavior, with its inherent nature and flaws, with its fallibility, is undoubtedly one of the key sources of hazards and - inevitably - of incidents and accidents. The surveillance and monitoring of human behavior is at the core of the so-called behavior-based safety (BBS), which" focuses on what people do, analyzes why they do it, and then applies a research-supported intervention strategy to improve what people do [4] and has to be seen as a discipline within the wider range of organizational behavior analysis [5]. A modern incident management approach that incorporates information gathering about any kind of hazard must also include data on all kinds of unsafe behavior or behavioral failures.

Having so far dwelled on WHAT to capture and process within incident management, we inevitably come to the next issues: HOW to ensure a near to complete capturing of all relevant information and WHOM to involve.

\subsection{Whom to involve?}

In the beginning of the history of safety management, safety was in the hands of line management. People managers were responsible for the safety of their coworkers, and general managers responsible for safe operations. The evolution of safety management techniques and methodologies brought the creation of the role of the safety engineer, the expert with respect to safety. Different safety roles were created, such as hazardous substance managers, industrial hygienists, safety engineers, and so forth. But still there was managerial responsibility for safety.

This has changed: with the rise of safety culture concepts and behavioralbased safety management, the individual employees have become the focus. This means that all workers should consider themselves responsible for their own and their co-worker's safety. Know-how about safety is spread more widely within the workforce to create a strong sense of common safety management. This is a collaborative safety approach.

As an example: companies with a very active safety culture promote keeping an open eye for safety-related issues within the corporation, to address safetyrelated issues, proactively address co-workers, and create awareness. Employees are more and more motivated to drive safe behavior and actively talk with their co-workers when they see latent dangers or unsafe behavior.

Modern incident management must consequently meet the needs that arise from this approach. Most important, any safety management concept has to include everybody in the company. Everybody has to become to a certain degree his or her own safety engineer. Everybody has to have access to the right information. Everybody has to have access to the right tools. This means, for example, that an incident management solution should have the tools for everyone uses to report hazards, near misses, and unsafe behavior. Consequently, these tools have to be tailored for non-expert users. They have to be tailored for occasional users. Usability and suppleness of an incident management 
application are crucial for user acceptance and for the successful setup of appropriate tools and processes.

With these requirements for successful incident management, we come without fail to a discussion of incident management within the context of an EHS strategy, with all its implications and dependencies.

\section{Incident management - in a wider context}

Incident management involves many stakeholders and aspects, from initial reporting to safety management to legal reporting. Any approach to setting up a successful incident management has to take into consideration the whole range of stakeholders and aspects. An isolated approach is bound to fail. It is therefore a crucial prerequisite to analyze incident management in the overall context of EHS and in the context of internal and external dependencies, within the corporation and outside.

\subsection{Incident management within EHS - a complex reality}

As mentioned above, it is important for the design and setup of a proactive safety culture driven by a modern incident management system to analyze the dependencies and interdependencies within the greater picture of EHS, with all its facets.

EHS has various centers of competence and activity; we might call them functional areas. And these functional areas - such as hazardous substance management (HSM), industrial hygiene (IH), occupational health $(\mathrm{OH})$, environmental compliance and reporting, and so on - have a great interdependency. There are process flows across and information flows between these areas. The various links between both the functional areas within EHS and between EHS and other units of the corporation are shown in the graph accompanying this section.

There is, first of all, the chain of links between HSM, IH, and OH. HSM deals with the materials that constitute a certain element of risk for the company, employees, and environment. The same materials become an integral part of the exposure profiles in $\mathrm{IH}$ as soon as people are exposed to them. The exposure profiles within the IH health risk assessments (HRAs) and information from HR together form the basis for medical protocol planning in $\mathrm{OH}$. There is also a clear link between waste management and HSM, since hazardous substances very often end up as waste, and this waste often has to be handled as a special form of hazardous substance. As such, it constitutes an exposure for co-workers involved in waste handling. Moreover, it is important to state that HSM is closely intertwined with the material flow within a company from the outset - from the substance's point of purchase until it leaves the company as a product, part of a product, or waste. Waste management is also connected to purchasing and material management to a certain extent in terms of the procurement of waste disposal services and the storage of waste. Furthermore, the financial dimension of waste disposal is also of crucial importance, since waste management costs 
contribute to overall material costs and may in fact influence purchasing decisions with respect to alternative substances. As a result, it is necessary to link this component with cost accounting and financial accounting. Waste management also incorporates a certain degree of environmental compliance (EC), such as legal reporting with respect to appropriate waste disposal.

The functional area of incident or accident management with hazard reporting/near-miss reporting incorporates a range of integration aspects. The aftermath of an incident often brings numerous other functional areas into play, including claims management, disability management, and return to work. Injuries and subsequent medical treatment necessitate the involvement of $\mathrm{OH}$, with information about the injuries and first-aid treatments that are needed as input for incident reporting. Of particular importance is the link between incident management and $\mathrm{IH}$ as a principal trigger of incident prevention, since the incidents, hazard reports, and near-miss reports are a key starting point for corrective actions and measures designed to make work areas safer. There is also an unavoidable link between the areas of disability management, $\mathrm{HR}$, and $\mathrm{OH}$. The reintegration strategies for co-workers with restrictions are compiled in cooperation with reintegration managers, $\mathrm{HR}$, and $\mathrm{OH}$ along with additional input from $\mathrm{IH}$, such as information about exposures in alternative work areas. HR itself is interlinked with any functional area in EHS related to the workforce - that is, any area that deals with safeguarding the employees. Parallel to this HR integration, there is also a link to third-party management, since whatever applies to in-house employees also applies to external employees such as contractors. It is also possible to draw a direct link between IH and plant maintenance as regards the corrective measures that have to be triggered as a follow-up to HRAs and incidents. When discussing incidents, it is impossible to ignore incident management for environmental compliance management. This is particularly true during a leakage, outlet, or spill that is harmful to the environment and subject to legal reporting obligations. With regard to regular emissions such as greenhouse gases, $\mathrm{CO}_{2}$, or other kinds of emissions subject to legal reporting, the necessary consumption information about input quantities needs to be obtained from purchasing or material management so the relevant emission figures can be calculated.

Finally, one particular interface has importance that cannot be overstated: the human interface. In any holistic EHS approach the "individual" must be considered as a key factor for the success of an EHS management system. Alongside expert users such as industrial hygienists, occupational health practitioners, and the like, there are many other stakeholders who need to be involved in EHS. There are various hierarchies of management, each with very different information requirements ranging from very condensed and exceptiontriggered C-level reporting down to a manager's view of his or her team members' exposures, $\mathrm{OH}$ planning, safety training, and so on.

Finally, of very particular importance, one has to consider all employees and contractors as a whole - everybody who is active in the company. It is a generally accepted fact that the top level of safety awareness can only be achieved by incorporating everybody into the EHS concept. In other words, 
everybody has to be a part of the EHS world. This can be achieved uniquely by involving all individuals, via self-service portals for EHS as well as employee information centers with information about exposures, safety regulations, personal protective equipment, planned and executed medical protocols, and so on. Transparency and inclusion are the first and most important steps toward achieving an excellent behavioral safety culture. And for a successful holistic EHS approach, it is essential to make the integration of people a core element of the EHS strategy from the very outset.

If we also take aspects of product safety and product compliance into account in such a concept, we would very likely discover even more interdependencies and interactions that would need to be added to the solution landscape setup for EHS.

A final aspect that further compounds this complexity is that most modern companies are part of a global economic system. Their customers, partners, and contractors are international. Similarly, most industries are interwoven into a global system of plants and subsidiaries in various countries and jurisdictions with differences in terms of language and culture.

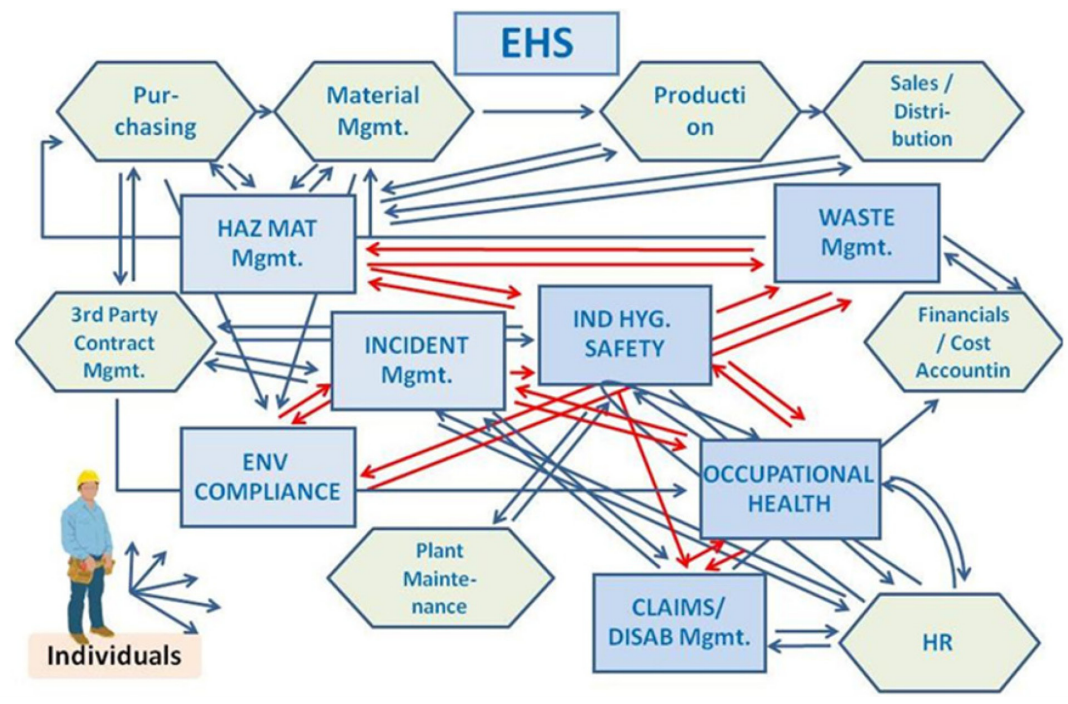

Figure 3: The complexity of EHS.

When seeking to design a successful global corporate EHS strategy that will work for all regions and adequately support all languages and local or regional requirements, it is imperative that this international or global aspect is given sufficient consideration. This is valid for the broader EHS view, but also for the consideration around the setup of a successful incident management as subset of the EHS strategy.

Essentially, all these aspects of interaction and interdependence point toward one crucial message - an isolated approach to any functional area of EHS is bound to miss the target. 


\subsection{Incident management within the corporate context}

Having come to that conclusion, it is clear that the next step must be to illuminate all facets of incident management, including all connections, dependencies and interdependencies with other functional areas or organizational units. And a wide range of those links are apparent.

The first and most obvious links are those to other functional areas within the world of EHS. Above all is the link to $\mathrm{OH}$ for first aid and the usual aftermath of accidents with employees injured. First-aid activity has to be triggered and reported on. But there is also the process of reintegration of injured employees with restrictions. For example, during the return-to-work process that follows an accident, $\mathrm{OH}$ practitioners are involved in close cooperation with HR, industrial hygienists, and incident managers.

With this we have automatically touched the area of HR and IH. The link with HR is very strong, reflecting the cooperation necessary with respect to people-related accidents. The same is also valid for contractor management, when third-party employees are involved in incidents or accidents. Industrial hygiene, with all its preventive activity, is based very much on the findings of incident management, especially in case of preventive proactive incident management.

Also very obvious is the link to HSM. In case of incidents or accidents, hazardous substances are very often involved.

For all activities around prevention, it is very important to have a clear picture of what hazardous materials with what characteristics are where within the company. And HSM also provides all the safety-relevant information about the materials, from protective equipment to first-aid treatment in case of accidents.

The link between asset management, fleet management, and maintenance is also rather perspicuous, first for the assets, machinery, vehicles, and such that are involved and for the subsequent repair activity. Additionally, with its preventive activity, IM drives maintenance activity around assets to improve their safety, by constructing protective barriers, using safety switches or speed delimiters, and so on.

Another important link is the connection with governance, risk, and compliance - the area of corporate risk management. It is important to be able to monitor hazards and incidents or accidents in incident management and to understand the connection between these actual occurrences and the risks that have been established and assessed within the corporate risk management, in order to control those risks and match them with reality.

Also very important is the smooth flow of information from incident management to top management, such as C-level, and to company representatives and legal units. If a major incident happens, the public is today informed in near real time. Nearly simultaneously with fire brigades and ambulances there are the first journalists and reporters on site. Company representatives and legal advisors therefore have to be in line with any major incident as quickly as first aid is called, to protect the corporation from 
inadequate statements and reactions. This is of vital importance to shield the company from avoidable legal persecution and to protect the brand name.

Another important facet of incident management is external and internal legal reporting. On one side, companies strive to have accurate internal monitoring (using dashboards) to monitor efficiency - in this case, efficiency of safety processes and incident/accident statistics. On the other side, there are various legal obligations, such as reporting injuries to legal bodies or insurance companies or informing environmental agencies about releases (such as giving information to water protection squads).

Last but not least for a company as economic unit is the financial aspect. What are the costs and collateral costs of accidents or incidents? What are the costs of preventive measures and safety activities? What amounts in compensation have to be paid for clearing water or ground after major incidents? What are the potential savings due to safety measures? Which organizational unit should be charged with the costs of incidents or accidents?

Summarizing, it can be said that incident management is not an island but highly intertwined with many units, processes, and activities within a corporation. An isolated approach, a point solution to fix some issue, or a standalone tool may cover some aspects to a certain degree, but any comprehensive approach to a proactive safety management with an active safety culture inevitably requires a flexible and highly integrated solution that can meet the needs and fit the capabilities and qualifications of very different stakeholders within a holistic solution landscape for EHS.

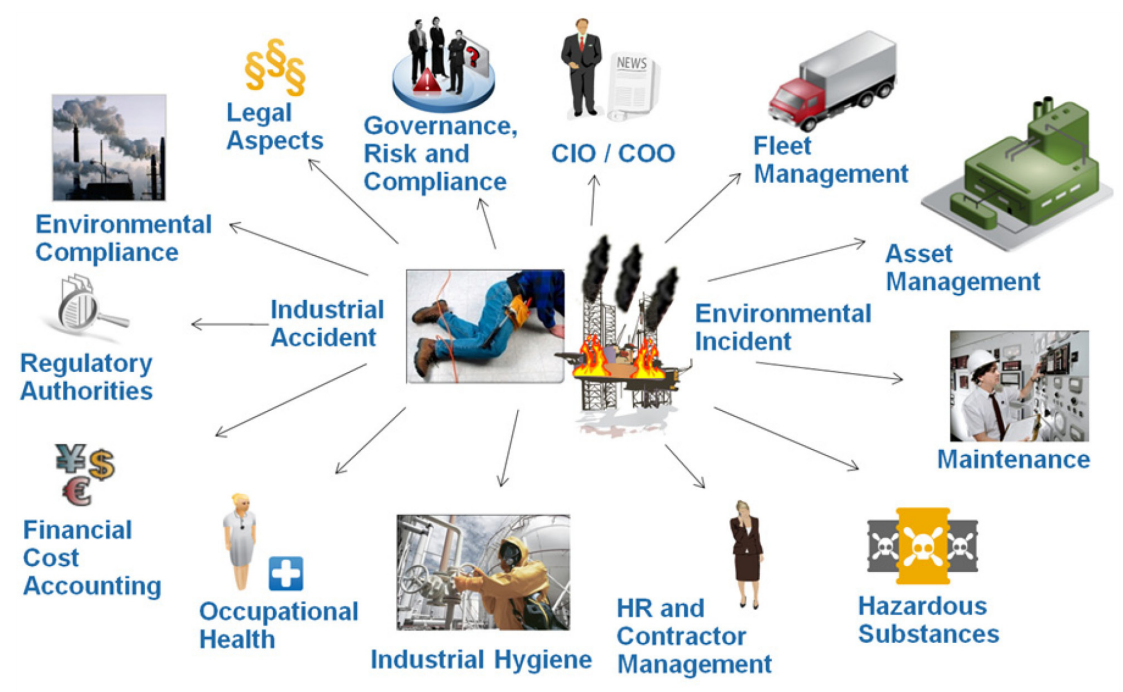

Figure 4: Stakeholders in incident management. 


\section{Incident management - the suitable way forward}

Anybody who faces the challenge of implementing an incident management solution in some way, including all the various aspects and facets of such a solution setup, will need a systematic approach or road map for the way forward.

A systematic approach can have different designs or granularities, but roughly there are five principal steps to keep in mind:

1 Problem analysis

2 Process design

3 Integration design

4 Solution setup

5 Flexible deployment

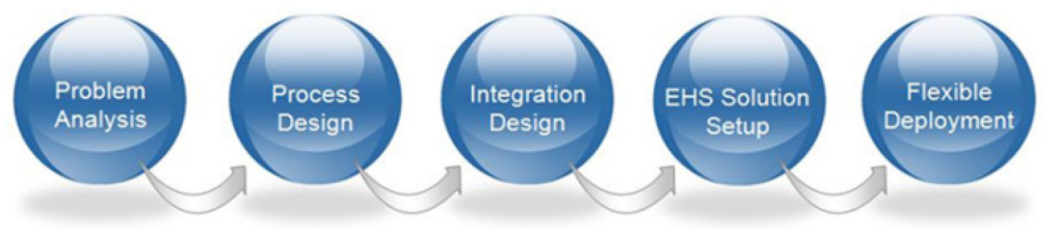

Figure 5: $\quad$ Phases of incident management implementation.

\subsection{Problem analysis}

First of all, perform a detailed and concise analysis of the status quo, of the present situation, of the challenges, and of the objectives that have been set for the new approach to incident management.

\subsection{Process design}

Based on step one, a detailed design should be done. As we've learned from the preceding sections, such a design should not focus on functions but rather on processes - processes that in many cases are cross functional and interdisciplinary. In case of incident management, this becomes apparent when drawing up the flow of action and information from first reporting until the final activities around investigation, prevention, and so forth are closed.

\subsection{Integration design}

Very quickly it also becomes visible where information is needed from which area - that is, where there are integration needs between the functional areas. The previously discussed complexity of an EHS solution landscape and the many facets of incident management have shown that a high degree of collaboration, and consequently system interaction, is an important factor for success. 


\subsection{EHS solution setup}

The next step is the setup of a system or of processes to facilitate the incident management activity. Given the many very different stakeholder groups around EHS and incident management and their very different needs, it is obvious that to be suitable and acceptable a solution must have a certain degree of user orientation, adaptability, and ease of use. Apart from the pure need for the processes, the usability of the solution and its acceptance by the users are key factors for success.

\subsection{Flexible deployment}

Last, but still of great importance, is the operation of the incident management solution, which should be driven as flexible deployment. A key factor of success for organizations is today to keep pace and sustain momentum as a learning organization.

The basis for this process of learning and improving is the ability to monitor success and efficiency. A successful incident management system has to first provide the tools and means to measure the success of the system (such as development of incident numbers and dashboards) but it must also have monitoring functionality to ensure the efficiency of the inherent processes and activities. Only based on this learning is it possible to drive improvement.

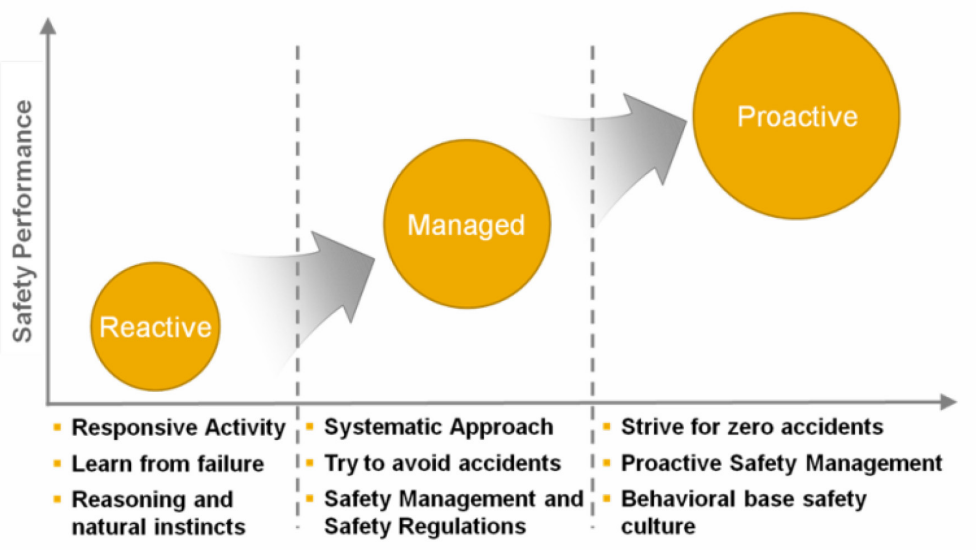

Figure 6: Maturity phases of companies.

Especially when a company is coming from a rather rudimentary safety organization with a rather reactive approach to safety management, it is important to have the necessary insight into the actual performance to drive the evolution, to come to a more systematic and managed approach, and in the final transformation step to create - based on experience and insight - a practiced safety culture and proactive safety management. 


\section{Conclusions}

Summarizing, it can be said that incident management is far more than an administrative process around incidents or accidents. On the contrary, it is a highly interactive and collaborative process, with many links and bridges to other organizational units and functional areas. And as such it has to be a kind of backbone of an active safety culture - an approach to safety that not only enables processes and functions but also facilitates evolution and progress with respect to the safety of the operation. It is the cornerstone for a learning organization with respect to safety, and the core prerequisite for the pursuit of a zero-accident environment.

\section{References}

[1] Heinrich, W. H.: Industrial Accident Prevention, 4th Edition. New York: McGraw-Hill Book Company, Inc., 1959

[2] Bird, Frank E., and George L. Germain: Loss Control Management: Practical Loss Control Leadership, Revised Edition, Det Norske Veritas (U.S.A.), 1996

[3] ConocoPhillips Marine: Safety Pyramid based on a study, April 2003

[4] Geller, E. Scott: "Behavior-based safety: a solution to injury prevention: behavior-based safety 'empowers' employees and addresses the dynamics of injury prevention." Risk \& Insurance, 2004

[5] Matthews, Grainne A.: "Behavioral Safety from the Consumer's Perspective: Determining Who Really Provides Behavior Safety." Cambridge Center for Behavioral Studies 